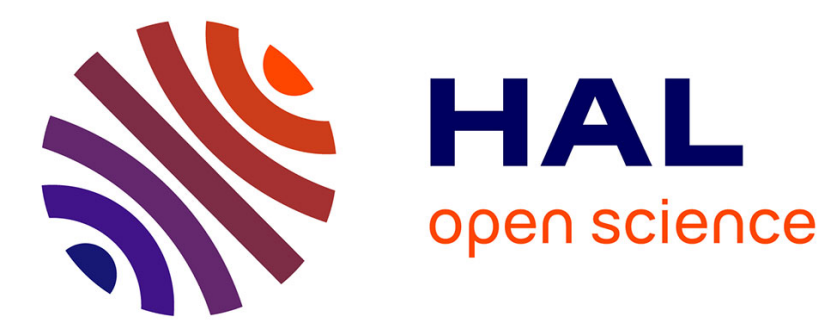

\title{
Boiling phenomena in near-critical SF6 observed in weightlessness
}

Carole Lecoutre-Chabot, Yves Garrabos, Daniel Null Beysens, Vadim Nikolayev, Inseob Hahn

\section{- To cite this version:}

Carole Lecoutre-Chabot, Yves Garrabos, Daniel Null Beysens, Vadim Nikolayev, Inseob Hahn. Boiling phenomena in near-critical SF6 observed in weightlessness. Acta Astronautica, 2014, 100, pp.22-29. 10.1016/j.actaastro.2014.03.012 . hal-00979046

\section{HAL Id: hal-00979046 \\ https://hal.science/hal-00979046}

Submitted on 21 Jun 2021

HAL is a multi-disciplinary open access archive for the deposit and dissemination of scientific research documents, whether they are published or not. The documents may come from teaching and research institutions in France or abroad, or from public or private research centers.
L'archive ouverte pluridisciplinaire HAL, est destinée au dépôt et à la diffusion de documents scientifiques de niveau recherche, publiés ou non, émanant des établissements d'enseignement et de recherche français ou étrangers, des laboratoires publics ou privés. 


\title{
BOILING PHENOMENA IN NEAR-CRITICAL SF 6 OBSERVED IN WEIGHTLESSNESS
}

\author{
Carole LECOUTRE, Yves GARRABOS \\ ICMCB-ESEME-CNRS - UPR 9048 CNRS, Université Bordeaux I - 87 avenue du Docteur Schweitzer, F 33608 PESSAC \\ Cedex, France. \\ Daniel BEYSENS, Vadim NIKOLAYEV \\ Service des Basses Températures, UMR-E CEA/UJF-Grenoble 1, INAC, Grenoble, France and ESEME, PMMH-ESPCI, 10 \\ rue Vauquelin, 75231 Paris Cedex 5, France. \\ Inseob HAHN \\ Jet Propulsion Laboratory, California Institute of Technology, CA 91109, USA.
}

\begin{abstract}
Boiling phenomena in the two-phase region of $\mathrm{SF}_{6}$ close to its critical point have been observed using the high-quality thermal and optical environment of the CNES dedicated facility ALI-DECLIC on board the International Space Station (ISS). The weightlessness environment of the fluid, which cancels buoyancy forces and favorizes the three-dimensional spherical shape of the gas bubble, is proven to be an irreplaceable powerful tool for boiling studies. To identify each key mechanism of the boiling phenomena, the ALIDECLIC experiments have benefited from i) the well-adapted design of the test cells, ii) the high-fidelity of the ALI insert teleoperation when long-duration experiment in stable thermal and microgravity environment are required and iii) the high repeatability of the controlled thermal disturbances. These key mechanisms were observed by light transmission and interferometry technique independently with two sample cells filled with pure $\mathrm{SF}_{6}$ at a near-critical density. The fluid samples are driven away from thermal equilibrium by using a heater directly implemented in the fluid, or a surface heater on a sapphire optical window. In the interferometry cell, the bulk massive heater distinguishes two symmetrical two-phase domains. The modification of the gas bubble shape is observed during heating. In the direct observation cell, the gas bubble is separated by a liquid film from the thin layered transparent heater deposited on the sapphire window. The liquid film drying and the triple contact line motion during heating are observed using light transmission. The experiments have been performed in a temperature range of $10 \mathrm{~K}$ below the critical temperature $T_{c}$, with special attention to the range $0.1 \mathrm{mK} \leq T_{c}-T \leq 3 \mathrm{mK}$ very close to the critical temperature. The unique advantage of this investigation is to provide opportunities to observe the boiling phenomena at very low heat fluxes, thanks to the fine adjustment of the liquid-vapor properties, (e.g. surface tension), by precise control of the distance to the critical point. We present the new observations of the gas bubble spreading over the heating surface which characterizes the regime where vapor bubbles nucleate separately and grow, as well as liquid drying, vapor film formation, triple contact line motion, which are the key mechanisms at the origin of the boiling crisis when the formed vapor film reduces the heat transfer drastically at the heater wall.
\end{abstract}

Keywords: Boiling phenomena, boiling crisis, critical heat flux, liquid-gas critical point, sulphur hexafluoride, microgravity experiment

Email addresses: lecoutre@icmcb-bordeaux.cnrs.fr (Carole LECOUTRE), garrabos@icmcb-bordeaux.cnrs.fr (Yves GARRABOS), daniel.beysens@espci.fr (Daniel
BEYSENS), vadim.nikolayev@espci.fr (Vadim NIKOLAYEV), inseob.hahn@jpl.nasa.gov (Inseob HAHN) 


\section{INTRODUCTION}

One of the most efficient ways of heat transfer is boiling phenomena of a liquid in contact with a solid heater. Such a heating process attracts strong attention from thermal engineers, especially when boiling crisis occurs, which may produce an irremediable damage of a heat exchanger via the heater melting. Indeed, boiling crisis is a transition from a regime where vapor bubbles nucleate separately on the heater wall to a regime where the heater wall is entirely covered by a continuous vapor film. When formed, the vapor film reduces the heat transfer drastically at the heater wall, due to the low gas thermal conduction. This transition phenomena appears when the heat flux exceeds a threshold value, called the critical heat flux (CHF). Understanding the physics of the CHF is thus essential in industrial heat exchanger design and thermal management.

One of the most established phenomenological approaches characterizing the boiling phenomena and the boiling crisis is based on the so-called Nukiyama curve [1, 2]. However the desired identification of the phenomena that trigger the boiling crisis process is complex. Experiments remain an essential way to descriminate the mechanisms governing the bubble formation and growth, the bubble detachment and/or spreading, as well as the vapor film formation and stabilization. Futhermore, the experimental conditions where the critical dryout occurs on Earth make a true challenge for any detailed analysis, especially when deeper understanding of the boiling crisis under the gravity acceleration field is essential. The main reason is the highly nonequilibrium nature of the boiling crisis, which makes impossible a study in steady state conditions. It can only be studied during the very short transient period where the crisis takes place, which enhances the experimental difficulty of collecting the pertinent data. Finally, not so many works have adressed this tedious task, using mainly visualization methods of the bubble spreading with high-speed video cameras (see for example [3]) or high-speed high-resolution infrared thermometry (see for example [4]).

The present work reports preliminary results of an experiment using a fluid near its liquid-gas critical point in microgravity conditions. The experiment overcomes the high instability of the process by taking benefits from the universal slowing down of the dryout and the cancellation of the buoyancy forces. This allows to perform space- and timeresolved experimental studies of the bubble spreading and vapor film formation, which are necessary for subsequent detailed analyses supporting theoretical and numerical developments. Indeed, near the liquid-gas critical point, the thermal diffusivity tends to vanish, requiring a longer time for all the diffusive equilibration processes. This entails the evaporation process and the bubble growth slowing down as approaching the critical point, with a related vanishing of the local fluid motion. In addition, the convergence of the liquid and gas densities, associated to the reduction to zero of the surface tension, implies a steep decrease of the CHF value, so that the boiling crisis can occur for small heat powers and small temperature gradients. All the general trends of these properties are satisfying the universal power-laws as a function of the temperature distance to the liquidgas critical point [5], which helps the data analysis. However such observations are impossible on Earth where the gravity flattens the interfaces and prevents formation of the spherical bubbles. This occurs because the capillary length tends to zero. One thus needs to perform such experiments in the weightlessness environment to keep the conventional bubble morphology and to cancel gravity-driven flows in the two-phase fluid (see for example [6, 7]).

Boiling has been studied in microgravity conditions, where an interesting phenomena has been initialy observed in a heated sulfur hexafluoride $\left(\mathrm{SF}_{6}\right)$ sample near its liquid-gas critical point, using the ALICE 2 - CNES facility on board the Russian MIR space station [8. During heating, the single bubble spread over the heater surface while approaching and crossing the critical point to reach the homeogeneous domain. The modeling and numerical simulation [9, 10, of the thrust of production of vapor can explain this gas spreading, also evidenced in a ground-based experiment where magnetic field gravity compensation (with residual gravity forces of order $0.02 g_{0}$, where $g_{0}$ is the constant acceleration on Earth's) was produced in a small volume of parahydrogen near its critical point.

Here we report new experimental observations approaching the gas-liquid critical point of $\mathrm{SF}_{6}$ on well-designed test cells [13], using the high-quality thermal and optical environment of the CNES dedicated facility ALI-DECLIC [11, 12] on board the ISS (see for detail [14]). We show that the key mechanisms - bubble spreading, liquid drying, vapor film formation, triple contact line motion - have been captured. That opens a new route for the future analyses with the objective to place the boiling crisis at zero gravity in the context of critical dynamics of non-equilibrium systems, as suggested by recent studies [15, 16, 17.

The paper is organized as follows. Section 2 presents the pressurized optical cells (labeled DOC for direct observation cell, and IOC for interferom- 

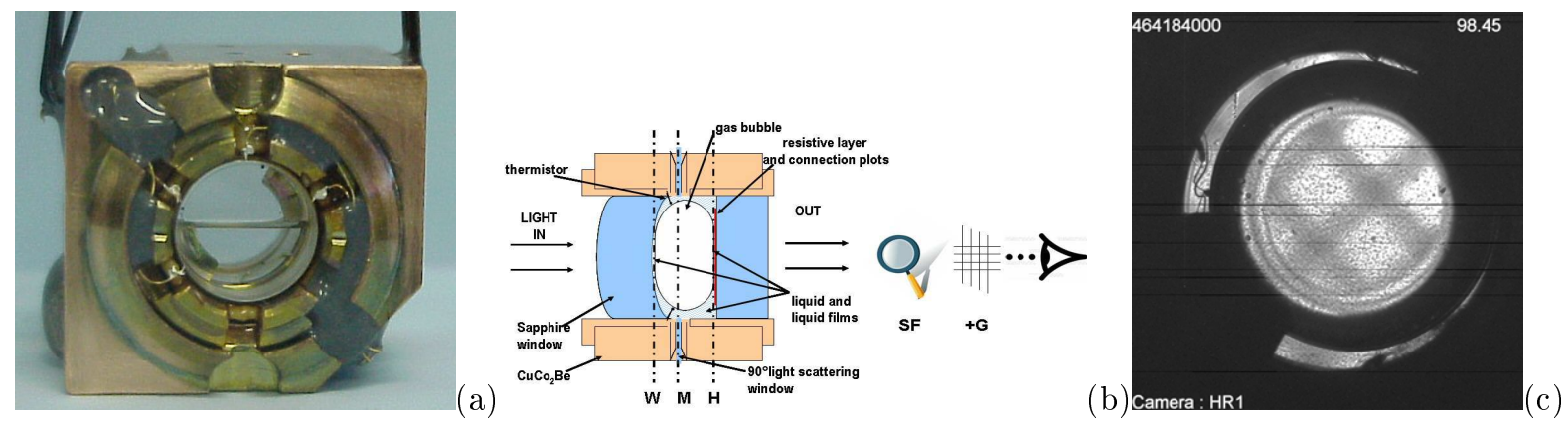

Figure 1: (a) Picture of direct observation cell (DOC) before integration. (b) Schematic cross section (not to scale) of the expected gas-liquid distribution in microgravity conditions. The transparent resistive layer $\left(\simeq 50 \mathrm{~mm}^{2}\right.$ area $)$ acts as a flat local heating source. (c) High-resolution video picture of the DOC in microgravity conditions at $T-T_{c}=-1 \mathrm{~K}$, observed using wide field light transmission with grid shadowscopy.

etry observation cell) designed to study the boiling crisis in non-homogeneous critical fluids. Sections 3 and 4 show main preliminary results obtained during 12 experimental sequences with the ALI-DECLIC insert, on one hand using the massive heater and interferometry observation technique (Section 3), and on the other hand the transparent resistive surface heater and light transmission methods (Section 4).

\section{Cell designs}

Pictures of the direct observation cell (DOC) and the interferometry observation cell (IOC) are shown in Figs. 1 (a) and 2(a), respectively. Each cell is filled with $\mathrm{SF}_{6}$ of electronic quality, corresponding to a 99.98\% purity (from Alpha Gaz - Air Liquide). The critical coordinates (temperature, pressure, density) of the gas-liquid critical point of $\mathrm{SF}_{6}$ are the following [18, 19:

$$
\begin{aligned}
& T_{c}=318.737 \mathrm{~K}, \\
& p_{c}=3.73 \mathrm{MPa}, \text { and } \\
& \rho_{c}=742.6 \mathrm{~kg} \mathrm{~m}^{-3} .
\end{aligned}
$$

The total fluid volume of DOC (IOC) is $0.468 \mathrm{~cm}^{3}$ $\left(0.508 \mathrm{~cm}^{3}\right)$, including a small dead volume mainly due to filling holes, corresponding to a total $\mathrm{SF}_{6}$ mass of $0.353 \mathrm{~g}(0.391 \mathrm{~g})$, leading to the filling mean density $\rho=\rho_{c}+1.6 \%\left(\rho=\rho_{c}+1.0 \%\right)$. The meandensity estimation of each cell uses a very precise optical method [20] based on the relative variation of the position of the liquid-gas meniscus on Earth, as a function of $T-T_{c}$.

\section{1. $D O C$}

The DOC design 13 leads to the expected microgravity configuration of the gas-liquid phase distribution given in Fig.1(b), where a central gas bubble is surrounded by the liquid wetting the cell walls and sapphire windows. The fluid sample volume mainly corresponds to a cylindrical volume of $D=$
$10.6 \mathrm{~mm}$ inner diameter and $e=4.115 \mathrm{~mm}$ inner thickness. Such a fluid sample configuration was indeed observed at large distance from $T_{c}$ using wide field light transmission, as illustrated in Fig. 1.(c).

The two main characteristics of this cell design are the following: (i) spherical concave surface of the entrance sapphire window $(\sim 0.3 \mathrm{~m}$ curvature radius); (ii) in situ integration of a heating device as a form of transparent resistive layer (labeled R8), deposited on the flat exit sapphire window [21. In the expected equilibrium configuration between two equal volumes of coexisting gas and liquid phases at critical density, the gas bubble is slightly compressed between the two sapphire windows, separated from the resistive flat layer by a thin liquid film, and maintained in symmetrical axial position by the concavity of the entrance window. These dimensional and shape requirements of the cell design are then appropriate for light transmission observation of the drying and the wetting of the liquid film that exists at equilibrium between the gas bubble and the resistive layer (see Fig. 1(c)). By exploiting optical microscopy (small field view $\sim 1 \times 1 \mathrm{~mm}^{2}$, corresponding to the central part of the wide field view $\sim 12 \times 12 \mathrm{~mm}^{2}$ ) and grid shadowscopy of the DECLIC-CNES instrument [12, we will show in Section 4 some characteristic video-pictures of the drying mechanism of the inserted liquid film, monitoring the transparent resistive layer as a local heating source.

The direct observation cell also allows to record the light scattered at small angles and at $90^{\circ}$. In addition, three small thermistors labeled R5, R6 and R7 (THERMOMETRICS B10) are located inside the fluid volume. Their positions (visible on the pictures of Figs. 1 (a) and (c)) are optimized to measure three local temperatures (not reported here) close to the gas-liquid meniscus. 

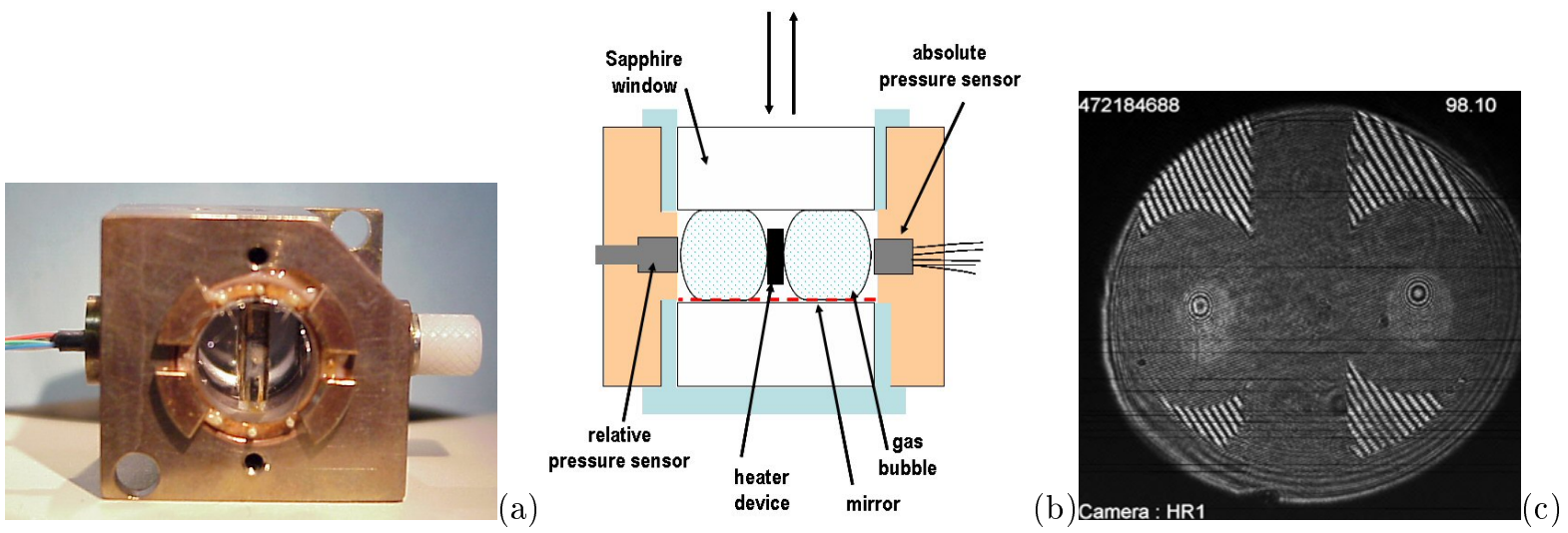

Figure 2: Picture of interferometry optical cell (IOC) before integration. (b) Schematic cross section (not to scale) of the IOC dedicated to study the bubble deformation due to boiling phenomena, using a central heater as a massive fluxmeter. (c) Interferometry observation of the two-phase $\mathrm{SF}_{6}$ sample in weightlessness, around $100 \mathrm{mK}$ below $T_{c}$; The experimental phase distribution is conform to the expected one in (b); The middle plate (dark) corresponds to a massive fluxmeter, which contains both a heating element and a temperature sensor (see text and Fig. 3 for detail).

\section{2. $I O C$}

The IOC is located in an arm of a Twyman-Green interferometer of the ALI-Insert of the DECLIC instrument [12. The IOC fluid volume mainly corresponds to a cylindrical volume of $D=10.6 \mathrm{~mm}$ inner diameter and $e=6.8 \mathrm{~mm}$ inner thickness. In a similar manner with the previous DOC case, a schematic design (not to scale) of this cell is given in Fig. 2(b). The expected configuration of the gas-liquid phase distribution in microgravity conditions is now represented as two symmetric gas bubbles surrounded by the liquid wetting the cell walls, the sapphire windows, and the walls of a massive heater. As illustrated by the interferogram picture given in Fig. 2. (c), the experimental phase distribution in microgravity is conform to the expected one in Fig. 2 (b). The middle plate (dark) corresponds to a massive fluxmeter, which contains both a heating element and a temperature sensor (see below and Fig. 3 for detail).

The main characteristic of IOC design concerns in situ integration of a heating device as a form of massive fluxmeter appropriate to interferometry observation of the shape deformation of the vapor liquid interface. The heater part of the fluxmeter is made from an assembly of four thermistors (labeled R3), while its central temperature is measured by a fifth central thermistor (labeled R2), as shown in Fig. 33(a). These thermistors are inserted within a massive copper block ( $8 \mathrm{~mm}$ length, $4.6 \mathrm{~mm}$ height, and $2 \mathrm{~mm}$ thickness), thermally isolated from the sapphire windows and the cell body [see Fig. 33 b)]. Comparing the interferograms of the heated twophase $\mathrm{SF}_{6}$ sample in ALI-DECLIC on Earth [see Fig. 3(c)] and on board the ISS [see Fig. 3(d)] il- lustrates the interest of the weightlessness condition for a better understanding of the boiling phenomena in near-critical experiments.

Two supplementary thermistors (labeled R1 and R4) are directly immersed in the fluid volume, respectively close to the central copper block [see Fig. 3.b)] and close to the cell wall (near the piezoresistive pressure sensor - see below). Moreover a piezoresistive sensor (ENTRAN EPB-B02-70B) monitored at high frequency $(2 \mathrm{kHz})$ can be used to measure the pressure inside the fluid, while a piezocapacitive sensor (KISTLER 6031), also monitored at high frequency, can be used to measure the relative variations of the pressure.

\subsection{General set-up}

These two experimental cells are housed in a Sample Cell Unit (SCU) whose temperature is monitored by a very accurate thermal regulation of the DECLIC facility, allowing $10 \mu \mathrm{K}$ of relative temperature accuracy and $40 \mu \mathrm{K} \mathrm{hour}^{-1}$ of temporal stability, in the range $36-60^{\circ} \mathrm{C}$. The sensors used for respective monitoring (label SCUr) and measurements (label SCUm) of the SCU temperature are made from high-stabilized platinum resistors $(100 \Omega$ resistance at $25^{\circ} \mathrm{C}$ ).

All the high-resolution and high-speed optical diagnostics are synchronized with in situ temperature and pressure measurements, and adjusted to the selected monitoring rate of the thermal stimuli produced by the two heaters (thin resistive transparent layer or massive fluxmeter). In addition, the heating period (from a minimum time of $43 \mathrm{~ms}$ ) is adjusted (from time increments of $43 \mathrm{~ms}$ ) in a synchroneous manner to the fluid temperature measurements and 

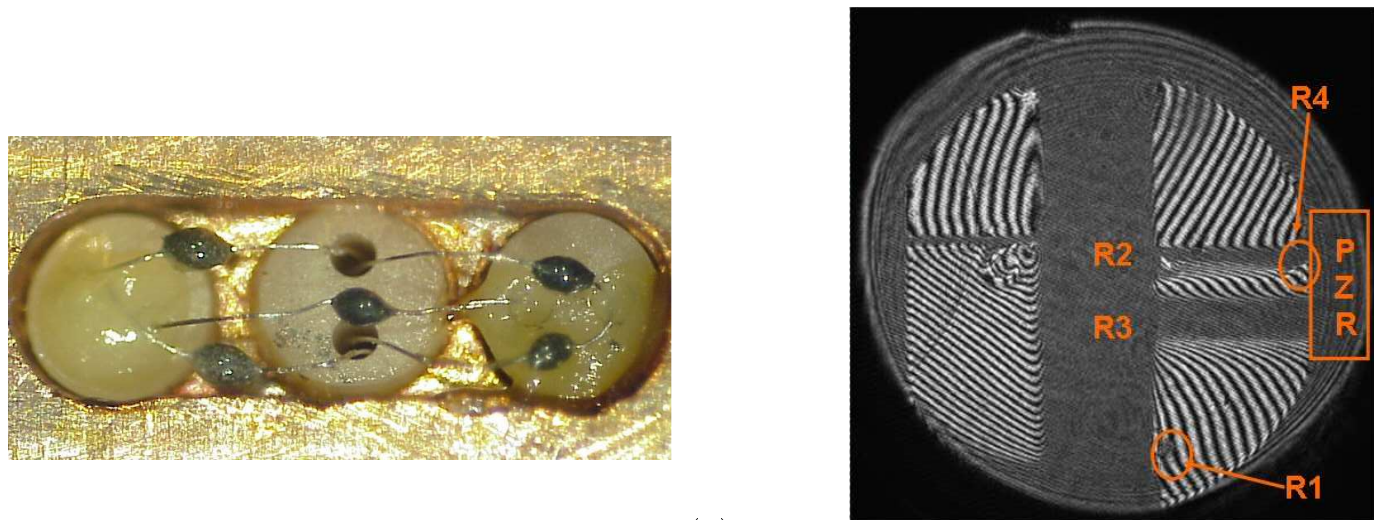

(a)
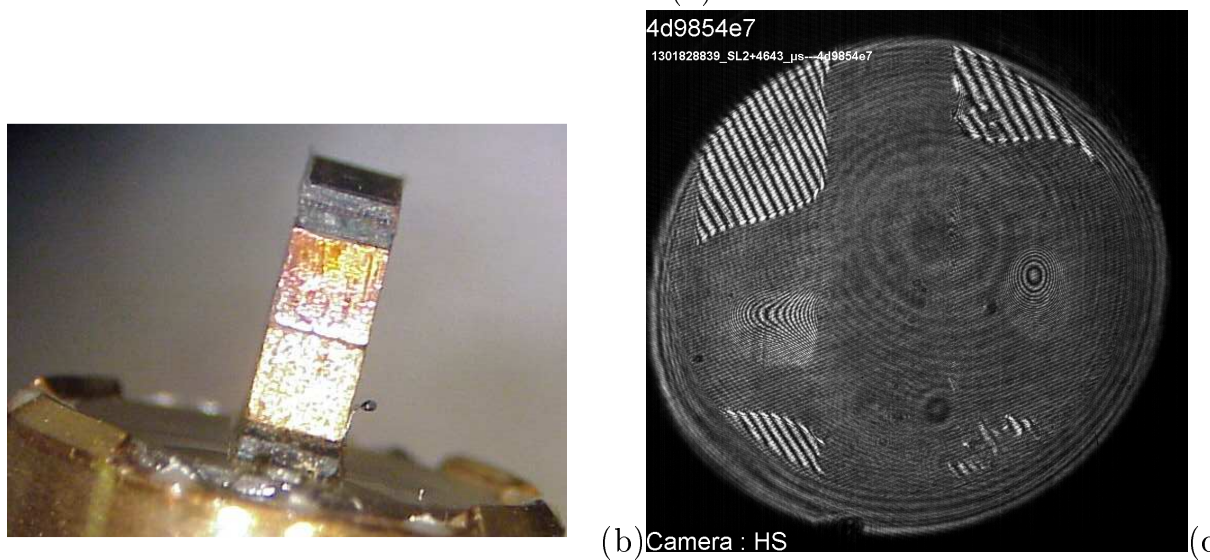

(d)

Figure 3: (a) \& (b): Details of the massive fluxmeter. (a) small thermistors inserted within the central part of a massive copper block of the fluxmeter; (b) Isolated fluxmeter above the entrance sapphire window before integration in the IOC body. (c) \& (d): Interferograms of the heated two-phase $\mathrm{SF}_{6}$ sample on Earth and in weightlessness; (c) Interferometry observation on Earth of the two-phase $\mathrm{SF}_{6}$ sample around $100 \mathrm{mK}$ below $T_{c}$, 2s after starting a heat pulse (10 mWpower; $10 \mathrm{~s}$ duration); The middle plate (dark) corresponds to a massive fluxmeter, which contains both a heating element (R3) and a temperature sensor (R2) (see text and Fig. 3.a) for detail). R1 and R4 thermistors immersed in the fluid are inside the colored circles. Convective flows are visible on the interferogram, while we also note that power supply of a piezoresistive sensor (PZR) is associated to thermal dissipation inside the liquid phase (illustrated by interferogram distorsions in the right part of the fluid sample); (d) Interferometry observation in microgravity of the two-phase $\mathrm{SF}_{6}$ sample at temperature $T=T_{c}-20 \mathrm{mK}, 2.8 \mathrm{~s}$. after a $15 \mathrm{~mW}$ heat pulse dissipated in R3 switched during $2.8 \mathrm{~s}$; The interferogram shows the bubble spreading near the wall of the IOC massive heater (see text).

the digital high resolution $(1000 \times 1000$ pixels $)$ or high speed (up to 462 frame $^{-1}$ ) video recording.

Boiling experiments in the two-phase cells were performed during 10 among the 13 experimental sequences of the ALI-DECLIC utilization on board the ISS, from March 2011 to February 2014. That corresponds to 237 (IOC) and 327 (DOC) experimental conditions covering the temperature range $10 \mathrm{~K} \geq T_{c}-T \geq 2.5 \mathrm{mK}$ (IOC) $\geq 0.3 \mathrm{mK}(\mathrm{DOC})$ and the heat power range from $1 \mathrm{~mW}$ to $30 \mathrm{~mW}$ (IOC) or $0.02 \mathrm{~mW}$ to $3 \mathrm{~mW}$ (DOC), with the heating period range from $1.13 \mathrm{~s}$ to $1800 \mathrm{~s}$ (IOC \& DOC).

Considering only the characteristic optical configuration for each cell, we have then reported the experimental observations which satisfy the following objectives:
1) To demonstrate the practical efficiency of the single bubble spreading due to the vapor recoil force when the boiling process takes place close to the wall of the massive heater in the IOC;

2) To illustrate the specific interest of the optical diagnoses in the detailed analysis of the mechanisms involved if the dryout of the liquid film on the transparent heater in the DOC.

\section{Bubble spreading near the wall of the mas- sive heater in the IOC}

Figures 4 show 3 selected images of the evolution at $t_{i}+43 \mathrm{~ms}, t_{i}+1.4 \mathrm{~s}$, and $t_{i}+2.8 \mathrm{~s}$ (left to right, respectively), of the one-side interferogram of the IOC at $T_{c}-T=20 \mathrm{mK}$, while a heat pulse $(15 \mathrm{~mW}$ power, 


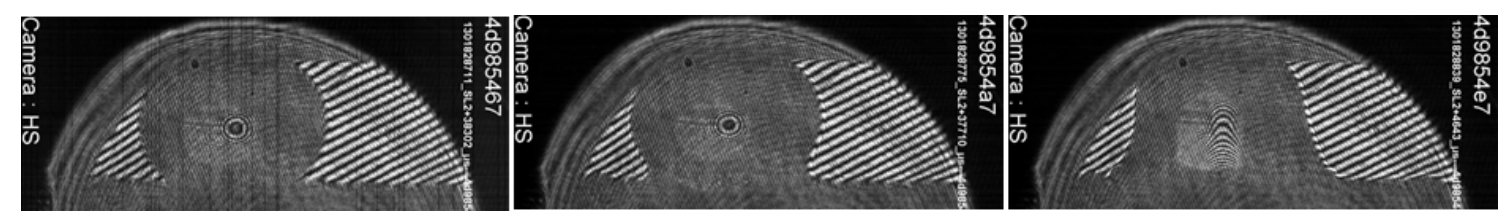

Figure 4: Sidewise observation of the single bubble spreading near the wall of the massive heater in IOC at temperature $T=T_{c}-20 \mathrm{mK}$. Left to Right: $t_{i}+43 \mathrm{~ms}, t_{i}+1.4 \mathrm{~s}$, and $t_{i}+2.8 \mathrm{~s}$. The heater is powered at $t_{i}=0 \mathrm{~s}$ with $15 \mathrm{~mW}$ dissipated in thermistors switched during $2.8 \mathrm{~s}$ duration.

$2.8 \mathrm{~s}$ duration) is powered into the massive heater. The high-quality of the images, combined with the high-symmetry properties of the initial shape of the confined single bubble in the well-designed IOC, gives access to the bubble spreading. Especially considering the median plane of the cell thickness, the superimposed deformation of bubble shape on the fringe pattern clearly demonstrates an increase of the apparent contact angle near the heating wall, which reflects the progressive heater dryout underneath the bubble. This latter phenomenon has been studied theoretically using the $2 \mathrm{D}$ cylindrical model of the single bubble. One of the most important parameters involved in these theoretical schemes seems to be the heater superheating, which measures the temperature deviation of the heater temperature from the saturation temperature of the two-phase equilibrium far from the heating wall. It was numerically predicted that the apparent contact angle grows with the heater superheating. In the specific limit of small superheating, it was also possible to formulate an analytic expression for the apparent contact angle.

We underline here that the collected data in such IOC near-critical experiments also provide the synchroneous local temperature measurements in the solid heater as well as in the fluid, to complement the video recording of the typical interferograms shown in Fig. 4. Therefore, we hope that the future data analyses of the IOC boiling experiments can address the estimation of the precise quantitative contribution of the heater superheating with the objective of testing the theoretical predictions of the apparent contact angle evolution.

\section{Dryout process near the layer of the trans- parent heater in the DOC}

\subsection{Dryout process of the thin liquid film oberved at large temperature distance from $T_{c}$}

Figures 5(a) to (f) show 6 wide field video pictures of the DOC at 6 different times $\left(t_{i}+0.5 \mathrm{~s}\right.$, $\left.t_{i}+4 \mathrm{~s}, t_{i}+8 \mathrm{~s}, t_{i}+9 \mathrm{~s}, t_{i}+14 \mathrm{~s}, \& t_{i}+20 \mathrm{~s}\right)$, during the thermal heat pulse $(3 \mathrm{~mW}$ power, $33.4 \mathrm{~s} \mathrm{du}-$ ration) supplied by the transparent heater powered on at $t_{i}=0 \mathrm{~s}$, at $1 \mathrm{~K}$-temperature distance below $T_{c}$. This figure illustrates the dryout process of the liquid thin film separating the confined gas bubble from the tranparent heater. We recall that the initial formation of this liquid film is due to the complete wetting at equilibrium in microgravity, whose initial extension is underlined by the grid shadowscopy observation and highlighted by a blue line in Fig. 5(a). At the early time of the heating, the film drying starts from the formation of a non-equilibrium triple contact line limiting the bulk liquid and the film liquid. Then, during the heat pulse, we observe the progressive drying of the central liquid film area through the evolution of a non-equilibrium triple contact line between the film liquid-gas-solid (see blue lines in Figs. 5 (b) to (e)). The complete liquid film drying is obtained after $\simeq 20 \mathrm{~s}$, as observed in Fig. 5(f).

We note the simultaneous bubble spreading indicated by the (non-equilibrium) bulk liquid-gas-solid contact line highlighted by a red line observed in Fig. 5 (b) to (f), clearly larger in size than the initial blue line observed in Fig. 5(a) (see above). This bubble spreading here observed at $T_{c}-1 \mathrm{~K}$ and already oberved in the IOC at $T_{c}-20 \mathrm{mK}$ (see previous section 3 ), remains also noticeable close to the critical temperature at $T_{c}-1.5 \mathrm{mK}$, as discussed in the next section.

\subsection{Initial dryout process of the bulk liquid and mo- tion of the triple contact line observed at small temperature distance from $T_{c}$}

Figures 6.a) \& (b) give two wide field video pictures of the DOC at two different times $\left(t_{i}+1 \mathrm{~s} \&\right.$ $\left.t_{i}+29 \mathrm{~s}\right)$ during the thermal heat pulse $(1 \mathrm{~mW}$ power, $33 \mathrm{~s}$ duration) supplied by the transparent heater powered on at $t_{i}=0 \mathrm{~s}$, at $1.5 \mathrm{mK}$-temperature distance below $T_{c}$. The low value of the surface tension at such a small temperature distance to $T_{c}$ authorizes a complex 3D-shape of the confined gas bubble at equilibrium saturation, which differs significantly from the previous one observed at $T_{c}-T=1 \mathrm{~K}$ where the centered $3 \mathrm{D}$-shape was governed by the concavity of the windows. Indeed, the initial two-phase distribution at $T_{c}-T=1.5 \mathrm{mK}$ shows that the confined gas bubble is now attached on the right part of 

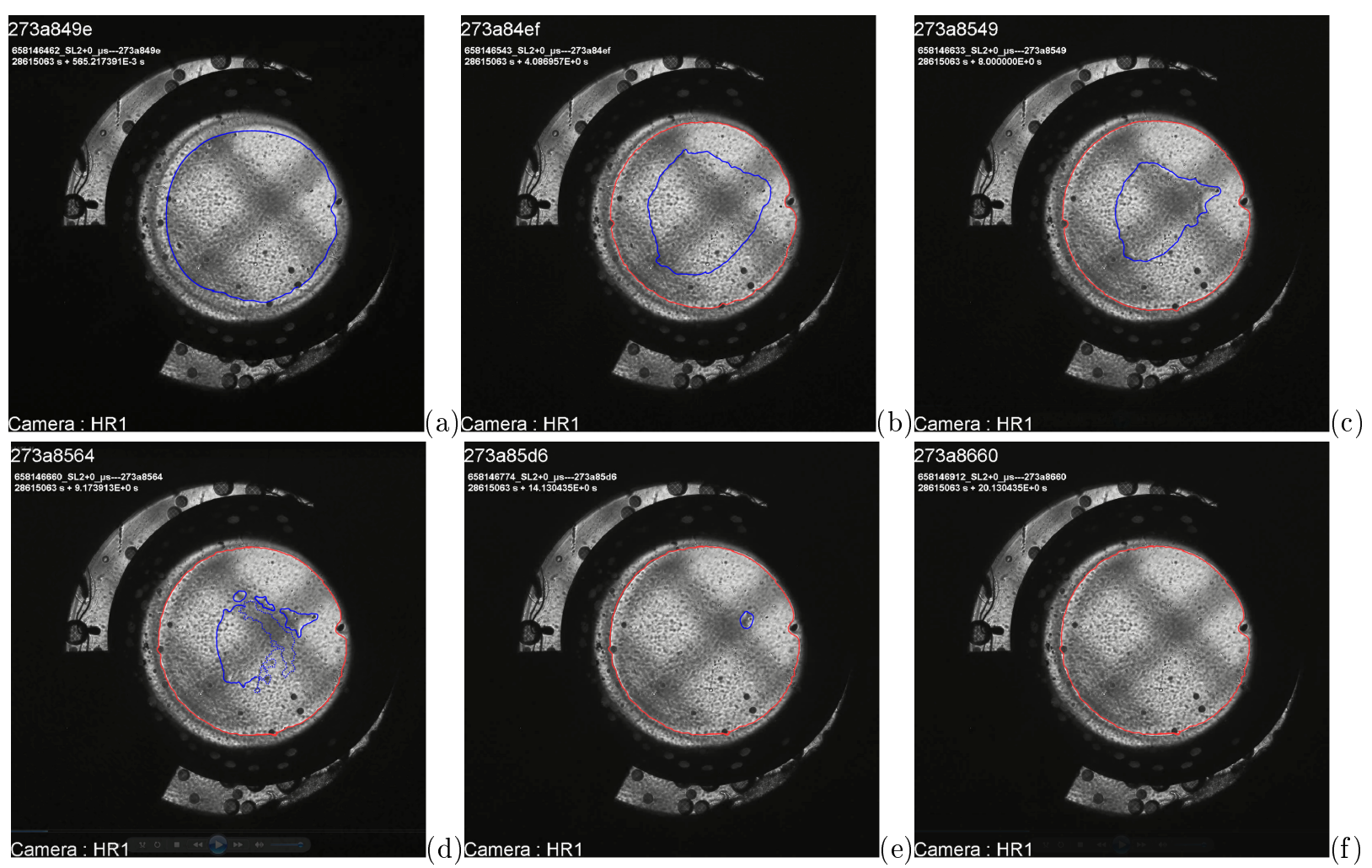

Figure 5: (a) to (f): Wide field video pictures of the DOC at 6 different times $\left(t_{i}+0.5 \mathrm{~s}, t_{i}+4 \mathrm{~s}, t_{i}+8 \mathrm{~s}, t_{i}+9 \mathrm{~s}, t_{i}+14 \mathrm{~s}, \&\right.$ $t_{i}+20 \mathrm{~s}$, respectively) during the thermal heat pulse $(3 \mathrm{~mW}$ power, $33.4 \mathrm{~s}$ duration) supplied by the transparent heater powered on at $t_{i}=0 \mathrm{~s}$, at $T_{c}-T=1 \mathrm{~K}$. The liquid film due to the complete wetting at initial equilibrium in microgravity corresponds to the inner area of the highlighted blue line (see video picture (a) and text). During heating, the increase of the liquid film drying area corresponds to the increasing area in between the (non-equilibrium) bulk liquid-gas-solid contact line, highlighted by a red line, and the film liquid-gas-solid contact line, highlighted by a blue line (pictures (b) to (e)). Video picture (f) corresponds to the complete drying of the liquid film when a spread gas bubble is in complete contact with the heater inside the area limited by the red line (see text).

the fluid sample volume due to a probable prominent pinning mechanism of the bubble on the right hole corresponding to the optical axis of the light scattering observation at $90^{\circ}$ (see Fig. 1). Such a distribution explains the (left) black curved line corresponding to the image of gas-liquid meniscus at the median position of the cell thickness. During the heating, this quasi-static position of this gas-liquid meniscus cannot be confused with the dynamic motion of the (non-equilibrium) triple contact line of the confined gas bubble close to the transparent heating layer. Indeed, close to the critical point the complete drying of the liquid film is quasi-instantaneous and only the (non-equilibrium) triple contact line between bulk liquid, gas, and solid is observed as a form of (right) white curved line (see Figs. 6(a) \& (b)). Moreover, these two video pictures also show the behavior of the dryout process occuring in the bulk liquid part in direct contact with the transparent heater (in the left side of the moving triple contact line).

Figures 6. c) \& (d) show two small field video pic- tures of the DOC where the (non-equilibrium) triple contact line between bulk liquid, gas, and solid is highlighted by a red line. The motion of this contact line at two different times $\left(t_{i}+1 \mathrm{~s} \& t_{i}+29 \mathrm{~s}\right)$ is clearly evidenced for a similar thermal heat pulse ( $1 \mathrm{~mW}$ power, $33 \mathrm{~s}$ duration) supplied by the transparent heater powered on at $t_{i}=0 \mathrm{~s}$. In the left part of the moving triple contact line we also clearly distinguish the behavior of the dryout process of the bulk liquid in contact with the transparent heater. From the DOC observations, a preliminary analysis of the contact line moving was reported in Fig. 7 as a function of time, at different $T_{c}-T$ values and for $3 \mathrm{~mW}$ and $1 \mathrm{~mW}$ power values. Maximum and minimum speeds were estimated about $110 \mu \mathrm{m} \mathrm{s}^{-1}$ and $20 \mu \mathrm{m} \mathrm{s}^{-1}$, respectively (see the corresponding blue lines in Fig. 7).

Finally, we note that the predicted increases of the apparent contact angle can be applied as a subgrid model to future $2 \mathrm{D}$ simulation of the bubble growth (with or without gravity). In such a simu- 

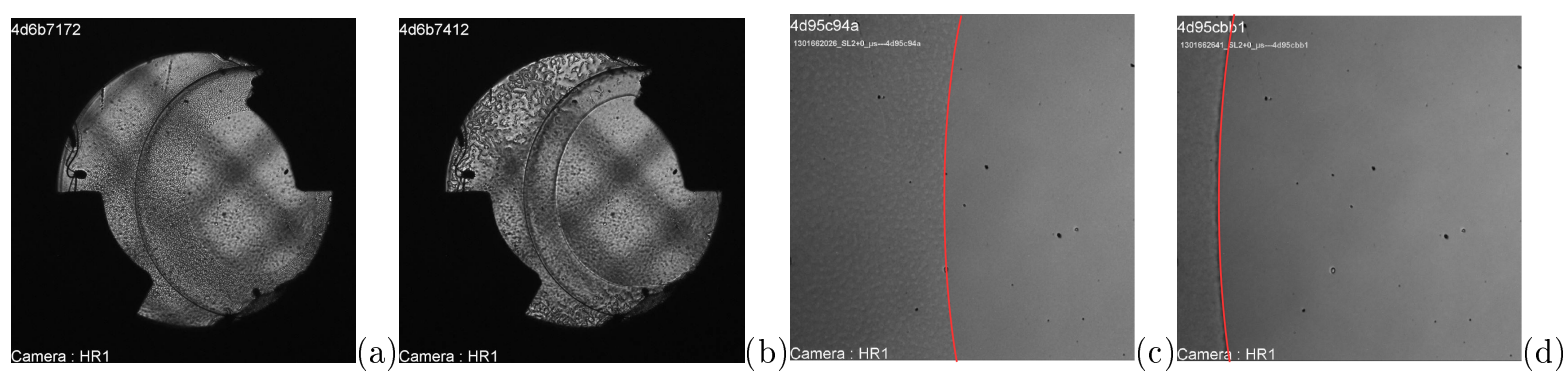

Figure 6: Video pictures the DOC in wide field ((a) \& (b)) and microscopy ((c) \& (d)) transmission, during a thermal pulse ( $1 \mathrm{~mW}$ power, $33 \mathrm{~s}$ duration) at $T=T_{c}-1.5 \mathrm{mK}$. Each observation is focused on the transparent resistive heating layer where the (non-equilibrium) triple contact line appears on the right part of the liquid-gas meniscus. The triple contact line is highlighted by a red line in microscopy video pictures (c) and (d). Complete spreading of the gas bubble is noticeable on the right side of the red line. The triple contact line motion is clearly distinguishable by comparison of the two similar pictures just after the pulse start $((\mathrm{a})$ and $(\mathrm{c}))$ and $29 \mathrm{~s}$ later $((\mathrm{b})$ and $(\mathrm{d}))$.

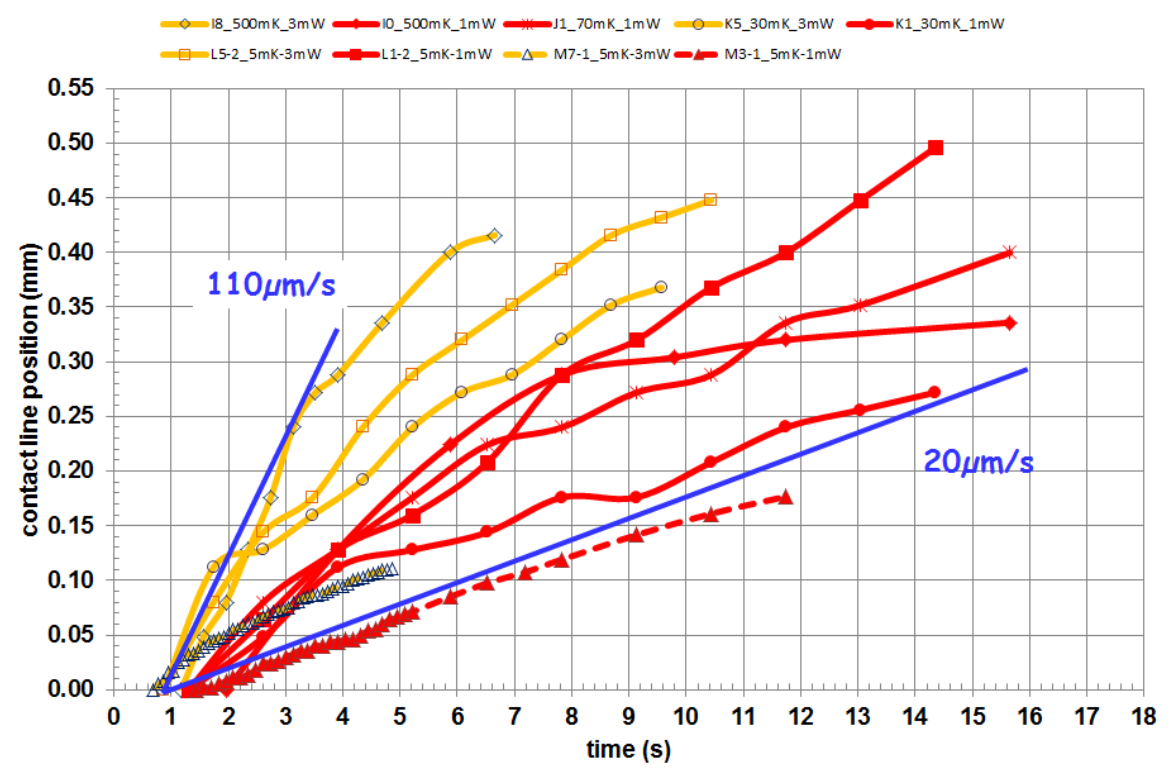

Figure 7: Preliminary results of the contact line moving for a representative set of heat pulses observed in the DOC during the ALI5-Seq7 run. Blue lines: preliminary estimations of the maximum and minimum speeds, respectively.

lation, the $90^{\circ}$ apparent contact angle can be postulated as a threshold value for the triggering of the boiling crisis. When this value is reached, it should be possible that the bubble coalescence becomes an efficient mechanism to create a continuous vapor film. Therefore, the preliminary observations of the bubble spreading and the dryout process reported here appear to be a first encouraging step towards a better understanding of the boiling crisis in microgravity.

\section{Conclusion}

Preliminary observations of the boiling phenomena in near critical experiments using ALI-DECLIC on board the ISS show that the expected key mecha- nisms of the boiling crisis have been captured during the heating of the well-designed sample cells filled with $\mathrm{SF}_{6}$. The main originalities of these new investigations, especially using an in-situ transparent heating device appropriate for light transmmission observation of the dryout process, is a combination of the fine monitoring of the injected low heat fluxes and the fine tuning of the liquid-vapor properties due to the fine control of the temperature distance to the critical point. We expect that the future analyses of the collected experimental data will provide definitive better understanding of the boiling crisis, especially focussing the objectives on the recently suggested zero-value of the CHF and critical behavior of the dry spot distribution in microgravity conditions, which places the boiling crisis in weightless- 
ness in the context of the critical dynamics of the non-equilibrium systems.

\section{Acknowledgments}

The authors acknowledge the financial support from CNES (Centre National d'Etudes Spatiales), and teleoperation capabilities from the CADMOSCNES user center in Toulouse, in collaboration with NASA support operating centers. They gratefully thank the CNES-DECLIC project team, especially project managers Gérard Cambon, Sébastien Barde and Gabriel Pont, and the associated DECLIC industrial teams (ASTRIUM-ST, COMAT, EREMS, IDEAS, SODERN, SEIV-Aquitaine, ARCOFLUID, AXS) for their help during the accomplishment of this work. VN also thanks the LMS team (Ecole Polytechnique) for the collaboration in the frame of the ALICE ANR-08-BLAN-0212-03 project.

\section{References}

[1] Nukiyama S., J. Soc. Mech. Eng. Jpn. 37, 367 (1934).

[2] Dhir V. K., Annu. Rev. Fluid Mech. 30, 365 (1998).

[3] Sakashita H. and Ono A., Int. J. Heat Mass Transf. 52, 744 (2009).

[4] Theofanous T. G., Tu J. P., Dinh A. T., and Dinh T. N., Exp. Therm. Fluid. Sci. 26, 793 (2002).

[5] See for example Anisimov M. A. and Sengers J. V., in "Equations of State for Fluids and Fluid Mixtures", Part I, Sengers J. V., Kayser R. F., Peters C. J., and White H. J., Jr., Eds. (Elsevier, Amsterdam, UK, 2000), pp. 381-434.

[6] Ma Y. and Chung J. N., Int. J. Multiphase Flow, 27, 1753 (2001)

[7] Straub J., Adv. Heat Trans., 35, 57 (2001).

[8] Garrabos Y., Lecoutre-Chabot C., Hegseth J., Nikolayev V., Beysens D., and J. P. Delville, Phys. Rev. E 64 051602 (2001).

[9] Nikolayev V., Chatain D., Garrabos Y., and Beysens D., Phys. Rev. Letters, 97, 184503 (2006).

[10] Nikolayev V., Chatain D., Beysens D. and Garrabos Y., Boiling crisis and evaporation at the triple contact line, ISPS 2007.

[11] Cambon, G., et al, 2004, 55th International Astronautical Congress, paper IAC-IAF/ IAA-04-J.5.03.

[12] Marcout R. et al, Proccedings of the IAC-2006, Valencia, Spain, 2-6 Oct. 2006, IAC-06-A2.5.02; see also http://smsc.cnes.fr/DECLIC/index.htm.

[13] Garrabos Y., Lecoutre-Chabot C., Beysens D., Nikolayev V., Barde S., Pont. G., and Zappoli B., Acta Astronautica, 66, 760 (2010).

[14] Pont. G. et al, Proccedings of the IAC-2012, Naples, Italy, 1-5 Oct. 2012, IAC-12-B3.4-B6.5.8 (2012).

[15] Janecek V. and Nikolayev V. S., Phys. Rev. E 87, 012404 (2013); Europhys. Lett. 100, 14003 (2012); Nikolayev V. S. and Janecek V., Int. J. Heat Mass Transf. 55, 7352 (2012).

[16] Janecek V., PhD thesis, Paris VI Univ. (2012).

[17] Lloveras P., Salvat-Pujol F., Truskinovsky L., and Vives E., Phys. Rev. Lett. 108, 215701 (2012).

[18] A. Kostrowicka a and J. V. Sengers, J. Chem. Phys. 111, 1551 (1999)
[19] Lecoutre C., Garrabos Y., Georgin E., Palencia F., Beysens D., Int. J. Thermophys. 30, 810 (2009).

[20] Morteau C., Salzmann M., Garrabos Y. and Beysens D. Proceedings of the Second European Symposium on Fluids in Space, Naples, Italy, april 22-26, 1996, pp. 327-333, A. Viviani (ed.) (1996).

[21] Y. Saadedin, PhD thesis, Bordeaux 1 Univ. (2007). 